\title{
MR Imaging Characteristics of Wingless-Type-Subgroup Pediatric Medulloblastoma
}

\author{
(D) Z. Patay, L.A. DeSain, S.N. Hwang, A. Coan, Y. Li, and D.W. Ellison
}

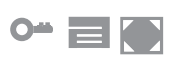

\begin{abstract}
BACKGROUND AND PURPOSE: "Transcriptionally different" medulloblastoma groups are associated with specific signaling pathway abnormalities; hence, they may present with distinct imaging manifestations. In this study, we sought to describe the MR imaging features of wingless-type-subgroup medulloblastomas with embryologic correlations.
\end{abstract}

MATERIALS AND METHODS: Pre- and postoperative imaging studies of 16 patients with wingless-type-subgroup medulloblastoma were evaluated for tumor location, involvement of surrounding CSF spaces or parenchymal structures, conventional and DWI signal properties, and postsurgical damage patterns. Laterality scores were assigned to tumors at each step in the evaluation process. Continuous variables were summarized by using descriptive statistics. The Wilcoxon signed rank test was performed to compare laterality scores. To determine the interobserver variability, we computed the intraclass correlation and Cohen $\kappa$ coefficients.

RESULTS: Wingless-type-subgroup medulloblastomas in our series were histopathologically "classic." Wingless-type-subgroup medulloblastomas occur in specific sites, with involvement of the foramen of Luschka (75\%), the fourth ventricle (68.75\%), the cisterna magna (31.25\%), and the cerebellopontine angle cistern (18.75\%). Laterality scores were low $(<2)$ when preoperative primary and secondary anatomic features were evaluated separately, but they increased $(>2)$ when all pre- and postoperative anatomic features were considered. Results were statistically shown to be reproducible (interclass correlation coefficient, 0.71-0.94; Cohen $\kappa, 0.63-1.00$ ). On the basis of anatomic lesion patterns, 4 location-based subtypes may be distinguished: 1) midline-intraventricular, 2) midline-extraventricular, 3) offmidline-intraventricular, and 4) off-midline-extraventricular, which represent a continuum.

CONCLUSIONS: Wingless-type-subgroup medulloblastomas are lateralized tumors arising from the brain stem and cerebellum around the foramen of Luschka. Our current understanding of their embryologic origins is in concordance with the spatial distribution of these tumors.

ABBREVIATIONS: $\mathrm{CP}=$ cerebellopontine; $\mathrm{L}=$ left; $\mathrm{LS}=$ laterality score; $\mathrm{R}=$ right; $\mathrm{SHH}=$ sonic hedgehog; $\mathrm{WNT}=$ wingless-type mouse mammary tumor virus integration site family

M edulloblastoma is a World Health Organization grade IV embryonal tumor occurring mainly, but not exclusively, in the pediatric population and is the most common malignant CNS tumor

Received November 24, 2014; accepted after revision April 13, 2015

From the Departments of Diagnostic Imaging (Z.P., L.A.D., S.N.H.), Biostatistics (A.C., Y.L.), and Pathology (D.W.E.), St. Jude Children's Research Hospital,

Memphis, Tennessee.

This work was supported in part by grant no. CA21765 from the National Cancer Institute and by the American Lebanese Syrian Associated Charities.

Please address correspondence to Zoltan Patay, MD, PhD, Department of Diagnostic Imaging, MS220, St. Jude Children's Research Hospital, 262 Danny Thomas Place, Memphis, TN 38105; e-mail: zoltan.patay@stjude.org

- Indicates open access to non-subscribers at www.ajnr.org

EIndicates article with supplemental on-line appendix and tables.

Indicates article with supplemental on-line photos.

http://dx.doi.org/10.3174/ajnr.A4495 in children. Beyond the traditional histopathologic classification, molecular subgroups based on gene-expression profiling have recently been recognized in these tumors. ${ }^{1-4}$ The subgroups, sonic hedgehog ( $\mathrm{SHH}$ ), wingless (WNT), group 3, and group 4, are biologically and clinically distinct disease entities, and distinguishing these groups in the clinical environment has become feasible. ${ }^{5-8}$

Studies suggest a distinct histogenesis for WNT, $\mathrm{SHH}$, and group 3 medulloblastomas. ${ }^{9}$ WNT tumors arise from the lower rhombic lip in the dorsolateral primitive brain stem, whereas $\mathrm{SHH}$ tumors arise from cerebellar granule neuron precursors in the upper rhombic lip. The different progenitor cell populations follow distinctly different migration tracts. These observations prompted us to hypothesize that WNT-subgroup medulloblastomas might present with distinct imaging characteristics, particularly in relation to spatial distribution in the posterior fossa. In this study, we report how the conventional MR imaging evalua- 
tion of WNT-subgroup medulloblastomas addresses this hypothesis.

\section{MATERIALS AND METHODS}

A retrospective institutional data base search was conducted with institutional review board approval and waiver of consent. Of the 238 patients $($ male $/$ female ratio $=1.74: 1$; age range $=0.23-22.4$ years) who were treated for medulloblastoma at our institution during a 10-year period (2000-2010), molecular subgrouping data were available for 143 (male/female ratio $=1.92: 1$; age range $=0.23-22.24$ years). Data obtained by immunohistochemistry, fluorescent in situ hybridization, and direct sequencing of formalin-fixed paraffin-embedded tissue showed that 16 (11.18\%) of these 143 patients had WNT-subgroup medulloblastomas $($ male $/$ female ratio $=1: 1$; age range $=6.47-14.53$ years; mean age $=9.82 \pm 2.18$ years). The initial diagnostic and first postoperative follow-up brain imaging studies of these patients were reviewed by using a standardized set of evaluation criteria. Fifteen patients had MR imaging studies available preoperatively; 1 had a CT only. All 16 patients had MR imaging studies postoperatively. Because many of the imaging studies were performed at referring institutions, imaging protocols were inconsistent. Preoperative, precontrast T1WI was available for 14 patients; T2WI, for 15 patients; FLAIR images, for 14 patients; and postcontrast T1WI, for 15 patients (as mentioned before, the only available preoperative imaging in 1 of the patients was precontrast CT). DWI data were available for 14 patients, but ADC map images were available for only 9. Review and evaluation of the imaging data were completed by 2 experienced pediatric neuroradiologists (reviewer 1 and reviewer 2). The second reviewer was requested to evaluate the preoperative and postoperative anatomic tumor features only to allow assessment of interobserver variability in the determination of the putative points of origin of the tumors. Including the second reviewer was to show that results related to tumor location features are reproducible.

Evaluation of Conventional MR Imaging Features of WNTSubgroup Medulloblastomas

Evaluation was completed by 1 reviewer and included the description of the tumor signal properties and other tumor features (cyst, necrosis, hemorrhage) by conventional MR imaging and DWI techniques. We estimated the volume of each tumor by using the sum of 3D, orthogonal tumor diameters. Additional detailed explanation of the methods used for the evaluation is provided in the On-line Appendix ("Methods 1").

\section{Preoperative Evaluation of Anatomic Tumor Features}

To evaluate the key anatomic tumor features (including the epicenter, hence the putative point of origin of the tumor), we adopted a stepwise, iterative evaluation process to create laterality scores (LS-1, LS-2, and LS-3), described in the On-line Appendix ("Methods 2").

\section{Postoperative Imaging Evaluation of Anatomic Tumor Features}

The next step of the evaluation process was focused on the abnormalities in the first available postoperative MR imaging study, which was typically performed shortly after near-total or gross-total tumor resection $($ mean $=5.3$ days, range $=1-18$ days). The rationale for this step was the assumption that visible (ie, T2 and/or FLAIR hyperintense) postsurgical lesions within cerebellar and/or brain stem parenchyma (ie, the postoperative damage pattern) reflect invasion of specific anatomic structures by the original tumor and provide clues to the epicenter (point-of-origin) of the tumor. Laterality scores (LS-4) were generated for the postoperative cerebellar and brain stem lesions (On-line Appendix "Methods 3"). We also recorded postoperative imaging evidence of any infarction in a PICA territory.

\section{Combined Pre- and Postoperative Evaluation of Location Features}

By using all available imaging data and the previously assigned laterality scores, a final LS (LS-5) was created for each tumor and was used to assign a consolidated score (LS-5c) for each patient (On-line Appendix "Methods 4").

The combined pre- and postoperative evaluation process is illustrated in Figs 1-4.

\section{Statistical Analyses}

Statistical analyses were performed by using SAS 9.3 statistical software (SAS Institute, Cary, North Carolina). Significance was assumed with $P<.05$. Continuous variables were summarized by using descriptive statistics, including number, mean, SD, and range; categoric variables were summarized by using number and percentage. The Wilcoxon signed rank test was performed to compare the laterality scores at different steps and stages of the imaging evaluation (eg, preoperative, postoperative). To determine the interobserver variability of laterality scores, intraclass correlation coefficients were estimated from a mixed-effects linear model analysis. The interobserver agreement of posterior fossa CSF-space involvement and tumor group assignment was examined by computing the Cohen $\kappa$ coefficient. Published guidelines were used to interpret intraclass correlation coefficients $(<0.40=$ poor; $0.40-0.59=$ fair; $0.60-0.74=$ good; and $0.75-1.0=$ excellent $)^{10}$ and Cohen $\kappa$ coefficients $(0-0.2=$ slight; $0.21-0.40=$ fair; $0.41-0.60=$ moderate; $0.61-0.80=$ substan tial; $0.81-1.0=$ almost perfect or perfect) ${ }^{11}$

\section{RESULTS}

All WNT-subgroup medulloblastomas in our series were histopathologically classic tumors. ${ }^{5}$

\section{Conventional MR Imaging Features}

All tumors for which precontrast T1WI was available exhibited hypointense signal. In T2WI, 6 (40\%) of 15 tumors were isointense compared with the cerebellar cortex, and the remaining 9 (60\%) were hyperintense. In FLAIR images, the signal properties of the solid tumor components were similar to the T2WI findings for all patients who had both T2-weighted and FLAIR images available. All tumors exhibited obvious hyperintense signal in diffusion-weighted images. In ADC images, 2 (22\%) of 9 tumors were hypointense, $6(67 \%)$ were hypointense+, and $1(11 \%)$ was hypointense ++ . In postcontrast T1WI, all 


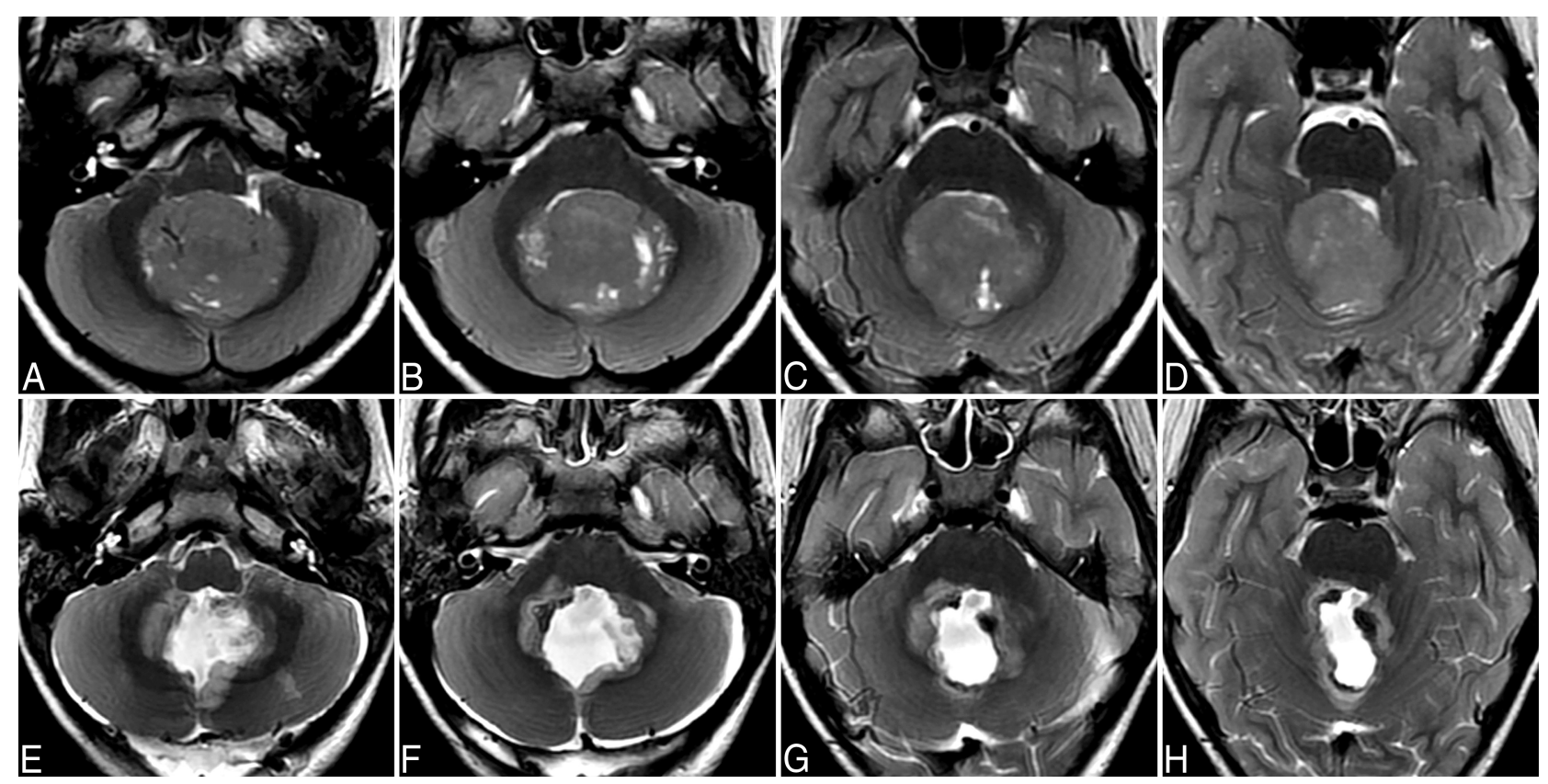

FIG 1. Pre- and postoperative transverse T2WI of a midline-intraventricular (subtype A) WNT-subgroup medulloblastoma. In the preoperative images $(A-D)$, fullness at the level of the right foramen of Luschka (LS-lc: RI) and right superior cerebellar peduncle (LS-2c: RI) suggests some laterality, yielding an LS-3c of R2. In the postoperative images $(E-H)$, the damage pattern is essentially bilateral, with bilateral involvement of the superior cerebellar peduncles (R1, LI), the dentate nuclei (RI, LI), inferomedial cerebellum (RI, LI), and tonsils (RI, LI). However the pattern shows some right-sided dominance, with unilateral involvement of the right pontine tegmentum (RT) and bilateral but dominantly right-sided involvement of the middle cerebellar peduncles (R2, L1). Although the tumor appears to be midline-intraventricular in the preoperative images, overall right-sidedness is suggested when all pre- and postoperative lesions and laterality scores are considered (LS-4c $=$ R2, LS-5c = R4).
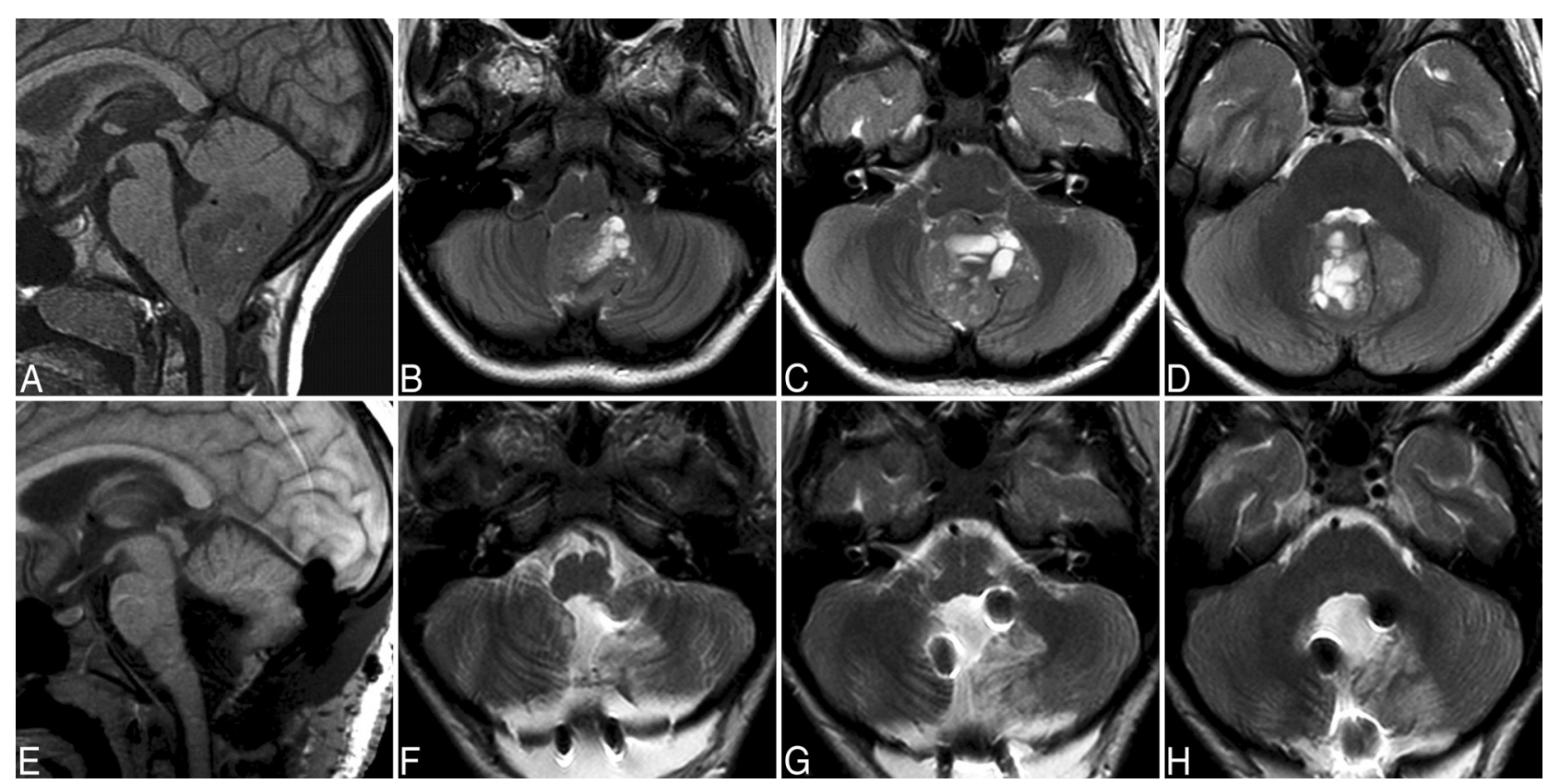

FIG 2. Pre- and postoperative sagittal TI-weighted and transverse T2-weighted images of a midline-extraventricular (subtype B) WNT-subgroup medulloblastoma. The preoperative sagittal Tl-weighted image $(A)$ shows a low-lying tumor in the posterior fossa, which is centered on the cisterna magna and pushing the vermis cranially, with extension to the foramen of Magendie but only minimal extension into the fourth ventricle. In transverse T2WI $(B-D)$, no definite laterality is seen; hence, the tumor appears to be gross midline, but there is some involvement of the left foramen of Luschka (LS- $\mathrm{lc}=\mathrm{LI}$ ) and left inferomedial cerebellum (LS-2c = L1). The combined preoperative laterality score is ambiguous ( $\mathrm{LS}-3 \mathrm{C}=\mathrm{L2}$ ). One of the PICAs (probably the left) is running over the upper aspect of the tumor, indicating displacement from below (D). Postoperative images $(E-H)$ show a relatively spared vermis and more pronounced abnormalities on the left side, which include damage to the left dentate nucleus and the left inferomedial cerebellum (LS-4c = L2) and a left PICA territory infarction. The combined pre- and postoperative laterality score suggests a left-sided tumor origin $(\mathrm{LS}-5 \mathrm{C}=\mathrm{L} 4)$.

tumors showed some signal enhancement; in 1 patient, it was faint; in 9 patients, it was moderate; and in the remaining 5 patients, it was avid. In $13(87 \%)$ of 15 patients, enhancement was seen within the entire tumor; in $2(13 \%)$ of 15 patients, smaller or larger areas of the solid tumor lacked perceptible enhancement and were labeled as inhomogenous. In another 6 


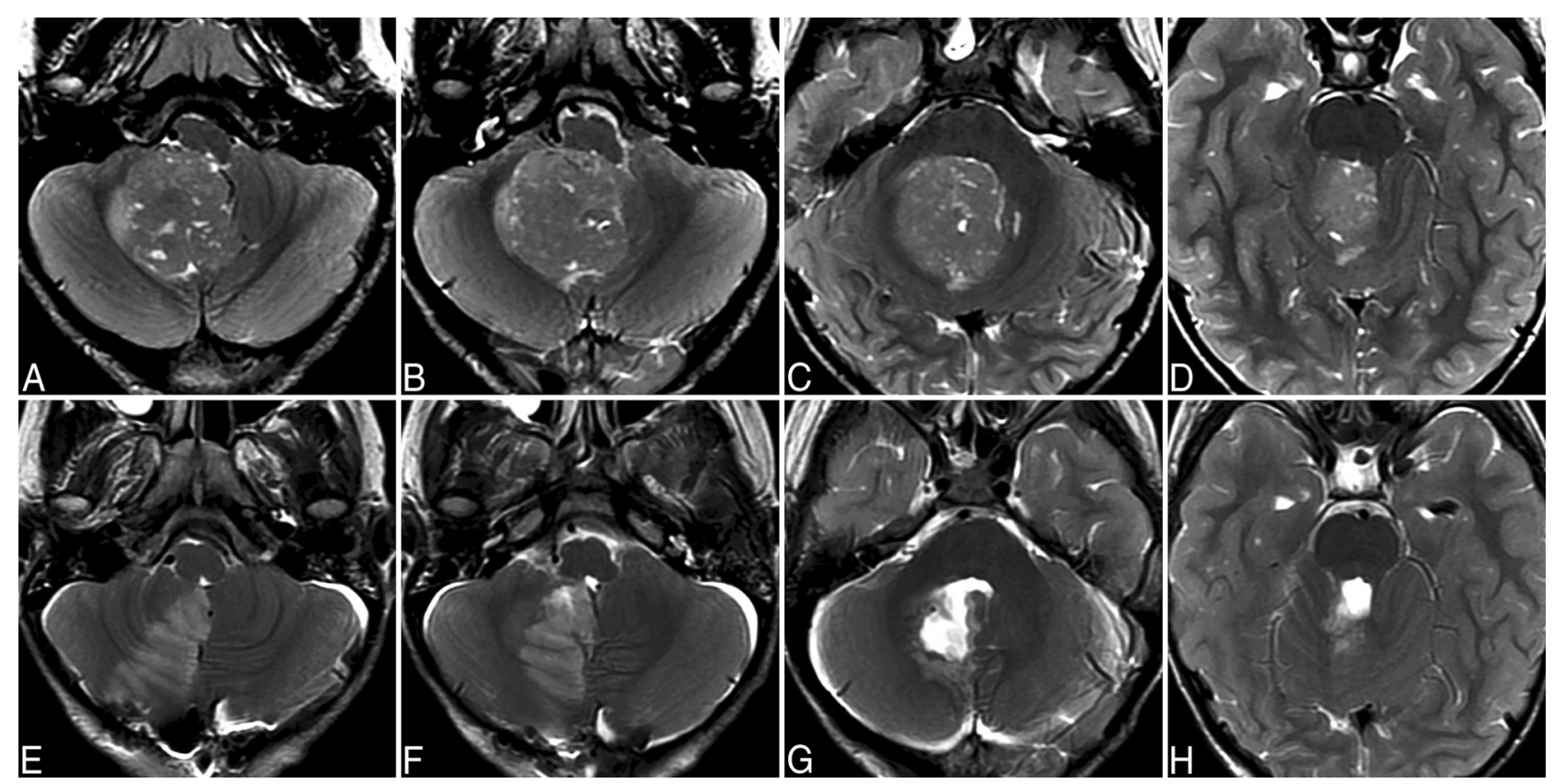

FIG 3. Pre- and postoperative transverse T2WI of an off-midline-intraventricular (subtype C) WNT-subgroup medulloblastoma. A right-sided predominance is evident in the preoperative images, but the tumor has a substantial intraventricular component $(A-D)$. This pattern is highlighted by the involvement of the right foramen of Luschka (LS-lc $=\mathrm{RI}$ ) and by invasion of the right dentate nucleus and right superior cerebellar peduncle (LS-2c = R2). Preoperative imaging data suggest that overall, this tumor is off-midline, with a right-sided predominance ( $\mathrm{LS}-3 \mathrm{C}=\mathrm{R} 3)$. The postoperative damage pattern is identical, with lesions in the right dentate nucleus and superior cerebellar peduncle $(\mathrm{LS}-4 \mathrm{C}=\mathrm{R} 2)$ and a right PICA territory infarction $(E-H)$. The aggregate laterality score indicates a clear right-sided laterality (LS-5C = R5).

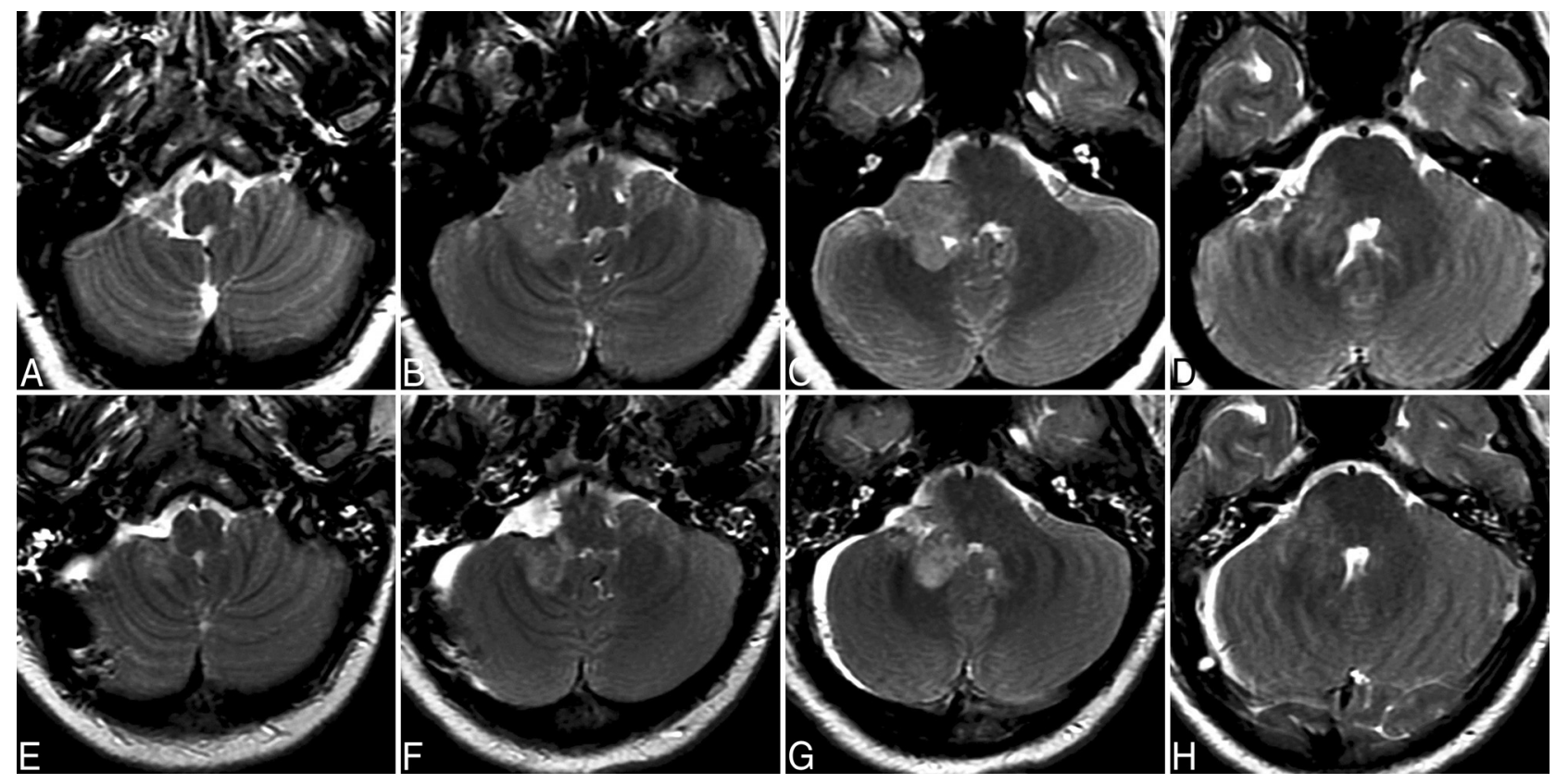

FIG 4. Pre- and postoperative transverse T2WI of an off-midline-extraventricular (subtype D) WNT-subgroup medulloblastoma. This tumor is centered on the right cerebellopontine angle cistern and involves the right foramen of Luschka $(B-C)$, with no extension to the fourth ventricle $(\mathrm{LS}-\mathrm{lc}=\mathrm{R} 2)$. Invasion of the right lateral brain stem and right inferomedial cerebellum is also suggested (LS-2c $=$ R2). Accordingly, preoperative anatomic imaging data indicate a definite laterality $(\mathrm{LS}-3 \mathrm{C}=\mathrm{R} 4)$. In postoperative images, damage is seen within the right lower brain stem $(F)$, right inferomedial cerebellum $(F-G)$, and right middle cerebellar peduncle $(H)(L S-4 c=R 3)$, with no damage to juxtaventricular structures (dentate nucleus, superior cerebellar peduncle). The aggregate laterality score indicates right-sided laterality (LS-5C = R7).

(40\%) of 15 patients, considerable signal inhomogeneities were found within the tumor field. In 7 (47\%) of 15 patients, the enhancement was homogeneous and involved the entire tumor. At least 1 tumor cyst was found in 7 (47\%) of 15 pa- tients. Some degree of intratumoral necrosis was noted in all except 2 patients. Evidence of intratumoral hemorrhage existed in $5(31.25 \%)$ of 16 patients; final diagnosis was MR imaging-based in 4 cases and CT-based in 1 case. 


\section{Pre- and Postoperative Anatomic Tumor Features}

These results reflect the findings of reviewer 1 . Observations by reviewer 2 were used to validate overall reproducibility (see results of the statistical analysis later in this section) and are not detailed here (but some are shown in On-line Tables 3-9).

All 16 WNT tumors appeared to be dominantly extraparenchymal and attached to the surface of the brain stem and/or the cerebellum. The posterior fossa CSF-space involvement is summarized in On-line Table 1.

Altogether, 36 distinct, secondary anatomic structures/sites were preoperatively found to be involved by tumor (range $=1-5$ / patient, mean $=2.2$ patient). The most commonly observed site of invasion was the dentate nucleus (11 [68.75\%] of 16 patients). In 9 patients, the invasion was unilateral (6 left, 3 right); in 2 patients, it appeared to be bilateral. The superior cerebellar peduncle was invaded in 5 patients, and the middle cerebellar peduncle, in 3 . One side of the inferomedial portion of the cerebellar hemisphere was apparently invaded by the tumor in $6(37.5 \%)$ of 16 cases: the lateral brain stem, in 4 cases (25\% overall, 3 on the right and 1 on the left); and the floor of the fourth ventricle, in the other 2 cases ( $12.5 \%$ overall, both on the left).

Altogether, 63 secondary anatomic structures showed postoperative imaging evidence of structural damage (range $=1-8 /$ patient, mean $=3.9 /$ patient). Of the 36 parenchymal invasion sites suggested preoperatively, 30 were confirmed in the postoperative images. Postoperative images showed 33 new lesions that were not recognized preoperatively (these did not include PICA territory infarctions); therefore, those 33 lesions were thought to represent collateral surgical damage.

\section{Laterality Scores}

The first consolidated laterality score (LS-1c), which was based on preoperative primary anatomic features, suggested some laterality (LS-1c: range $=1-2$ ) in the tumors of 11 patients $(68.75 \%)$ and showed no laterality (both LS-1 and LS-1c: 0 ) or uncertain laterality (LS-1: R1, L1, hence LS-1c: 0) in those of the remaining 5 patients $(31.25 \%)$. The mean of LS- $1 \mathrm{c}$ was $0.94 \pm 0.77$. The second consolidated laterality score (LS-2c), which was based on preoperative secondary anatomic features, suggested laterality in the tumors of all patients (LS-2c range $=1-5$, mean $=2.00 \pm$ 1.15). The third consolidated laterality score (LS-3c), which was based on combined preoperative primary and secondary anatomic features, showed even stronger laterality in the tumors of all patients (LS-3c range $=1-6$, mean $=2.94 \pm 1.53$ ).

Considering the specific CSF-space involvement pattern in conjunction with LS-3c, we established a group assignment for each tumor based on gross location categories (intra- versus extraventricular, midline versus off-midline). This categorization was based on 2 assumptions: 1) for individual tumors, any LS of 0-2 was weak and would not indicate definite laterality, but any LS of 3 or higher was strong and indicative of laterality; and 2) involvement of the fourth ventricle (with or without involvement of other CSF spaces) would indicate an intraventricular tumor. According to this scheme, 6 tumors were midline-intraventricular (subtype A), 2 were midline-extraventricular (subtype B), 5 were off-midline-intraventricular (subtype C), and 3 were off-midline-extraventricular (subtype D) by reviewer 1 . There was per- fect agreement between reviewer 1 and reviewer 2 in subgroups B and $\mathrm{D}$ and some disagreement in subgroups $\mathrm{A}$ and $\mathrm{C}$; reviewer 2 actually saw stronger laterality than reviewer 1 (On-line Tables 3-8). The ratio between apparent midline tumors (subtypes A and $\mathrm{B}$ ) and off-midline tumors (subtypes $\mathrm{C}$ and D) was 1:1 (and was 1:1.7 for reviewer 2). For examples, see Figs 1-4.

LS-4c, which was based on the postoperative damage pattern, suggested laterality (LS-4c: range $=1-6$ ) in the tumors of 15 patients $(93.75 \%)$ and was ambiguous (eg, LS-4 of R3, L3) in the 1 remaining patient $(6.25 \%)$. The mean of LS-4c was $3.06 \pm 1.48$. LS- $5 c$, which was based on all (pre- and postoperative) anatomic lesion data, showed tumor laterality in all patients (LS-5c: range $=$ $1-8$; mean $=4.69 \pm 2.09$ ).

Overall, LS-2c was significantly greater than LS-1c; LS-3c was significantly greater than LS-2c, and LS-5c was significantly greater than LS-4c. However, LS-4c was not significantly different from LS-3c (On-line Table 2).

The mean of the sum of the 3D tumor measures was $11.68 \pm$ $1.83 \mathrm{~cm}$, $($ range $=7.4-15.1 \mathrm{~cm})$ for the entire cohort. The largest tumors were found in the midline-extraventricular group (mean sum of orthogonal diameters $=12.7 \pm 1.27 \mathrm{~cm}$ ); but on average, off-midline-intraventricular tumors were only slightly smaller $($ mean $=12.48 \pm 1.75 \mathrm{~cm})$. The size of midline-intraventricular tumors was close to the mean size of the tumors of the entire cohort $($ mean $=11.53 \pm 1.43 \mathrm{~cm})$, and off-midline-extraventricular tumors were the smallest $($ mean $=9.97 \pm 2.42 \mathrm{~cm})($ On-line Tables 3-7).

PICA territory infarction was found in 4 patients (25\%). Three of these instances corresponded to an ipsilaterally positive LS (LS-4c values of 1, 2, or 3; LS-5c values of 3, 4, or 5); the other occurred without definite laterality in the postoperative damage pattern (LS-4c: 0) or the overall lesion involvement pattern (LS$5 c: 1$ ). Two of those cases involved tumors of subtype B; 1 , of subtype A; and 1, of subtype C.

\section{Interobserver Variability and Agreement}

There was "substantial" to "perfect" agreement among the 2 neuroradiologists in determining posterior fossa CSF-space involvement (Cohen $\kappa$ range $=0.63-1.00$; On-line Tables $3-7$ ). There was "substantial" agreement among the 2 neuroradiologists for the location-based "group assignments" (subtypes A-D) of each tumor (Cohen $\kappa=0.65$, On-line Table 8 ). All laterality scores showed "good" to "excellent" consistency between 2 neuroradiologists (intraclass correlation coefficient range $=0.69-0.94$; On-line Table 9). Overall, the results were shown to be reproducible, with acceptable interobserver agreement.

\section{DISCUSSION}

In this article, we describe the conventional MR imaging features and common locations of WNT-subgroup medulloblastomas by using an MR imaging-based descriptive approach to analyze preand postoperative images. Our results suggest that WNT-subgroup medulloblastomas are close to the midline yet are lateralized tumors originating from structures around the foramen of Luschka (superficial layers of the dorsolateral brain stem under the middle cerebellar peduncle and paramedian structures of the inferomedial cerebellar hemispheres). These findings advance 

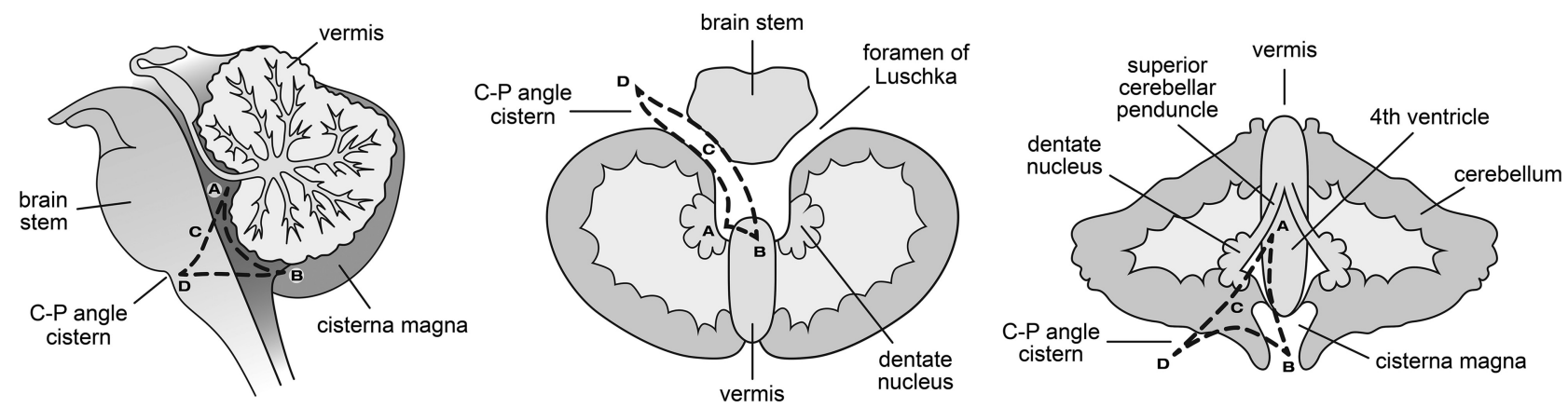

FIG 5. Putative points of origins of WNT-subgroup medulloblastoma in sagittal, transverse, and coronal planes. Letters $(A-D)$ indicate the theoretic epicenter of tumors in the different subtypes; however, the points of origin represent a continuum, and actual tumors may be centered anywhere along the dotted lines.

our current understanding of the implications of tumor location in molecular medulloblastoma subgroups and correlate well with recent discoveries about the developmental origins of WNT-subgroup medulloblastoma and pertinent aspects of the WNT molecular signaling mechanism., ${ }^{9,12}$

In the human embryo, the cerebellum, pons, and medulla oblongata develop from the primitive rhombencephalon. The rhombencephalon is initially formed by 8 rhombomeres, which are present by the end of the fourth fetal week of life, but the development of the cerebellum, in particular, continues even after birth. Each rhombomere has specific sets of transcription factors and receptors, and specific progenitor cell populations have been identified within each rhombomere, which give rise to various cell populations that form the future cerebellum or brain stem. $\mathrm{SHH}$ tumors originate from glutamatergic granule cell neuron precursor cells, which are derived from the upper rhombic lip. Conversely, WNT tumors originate from cells arising from the lower rhombic lip. ${ }^{9}$ The latter normally migrate along the anterior precerebellar extramural migratory stream (AES) to form nuclei within the posterior portion of the brain stem, but mutant cell populations may give rise to tumors such as WNT-subgroup medulloblastomas in childhood. WNT pathway derangements (somatic mutations of the $\beta$-catenin gene, CTNNB1) have been found in up to $10 \%$ of sporadic medulloblastomas, and WNT tumors represented approximately $11 \%$ of our cohort with available molecular data. ${ }^{4,13}$

Conventional and DWI features of WNT-subgroup medulloblastomas are quite similar to those described in the literature. ${ }^{14,15}$ Consistent with the high cell attenuation in embryonal tumors of the CNS, WNT-subgroup medulloblastomas have lower T2 signal than do other common pediatric posterior fossa tumors, such as juvenile pilocytic astrocytomas, ependymomas, and choroid plexus tumors. Our data suggest that the diagnostic value of precontrast T2WI and FLAIR imaging is not substantially different. The only potential diagnostic benefit of precontrast FLAIR imaging may be its ability to distinguish a tumor cyst and a sequestrated fourth ventricle space (both may appear quite similar in T2- and T1WI); but in clinical settings, this advantage may not affect disease management enough to justify the additional examination time. DWI remains the most valuable technique in the differentiation of medulloblastomas and all other nonembryonal tumors in the posterior fossa. It invariably shows moreor-less restricted diffusivity within solid tumor areas with corre- sponding low signal in ADC map images. Although quantitative analysis of ADC data could not be performed in our cohort, variations in ADC signal were recognized, but their significance is still unclear. Because all of our patients had histopathologically classic medulloblastoma, specific correlations between radiologic and histopathologic data were not sought in this cohort. Previous attempts at making such correlations have led to limited or controversial results. ${ }^{16,17}$

Regarding the spatial distribution of WNT-subgroup medulloblastomas in the posterior fossa, our study provides new insights into the most common sites of predilection more precisely than is described in a recent report, ${ }^{18}$ which found that $75 \%$ of WNT-subgroup tumors occurred along the cerebellar peduncle and the cerebellopontine (CP) angle cistern. Our findings are concordant in that $\mathrm{CP}$ angle and foramen of Luschka locations (subtypes C and D in our spatial classification scheme) are highly characteristic of WNT tumors, but we found that the "off-midline" subtypes account for only $50 \%$ of the cases. In our experience, WNT tumors seem to develop along an oblique-curved triangle centered on the foramen of Luschka, with 1 peak extending ventrolaterally to the $\mathrm{CP}$-angle cistern, another posteroinferomedially to the cisterna magna, and the third posterosuperomedially to the fourth ventricle (Fig 5).

Consequently, MR imaging shows many of these tumors as clearly off-midline (subtypes C and D) and others as apparently midline (subtypes A and B). To investigate putative points of origin of the latter 2 subtypes, we developed an iterative evaluation process that takes into account pre- and postoperative imaging features indicating tumor invasion of specific anatomic regions and structures. The resultant LSs suggest laterality even in the apparent midline cases; therefore, we speculate that midline-intraventricular WNT-subgroup medulloblastomas may originate near the ventricular orifice of the foramen of Luschka (eg, lateral recess of the fourth ventricle) and spread upward along the lateral wall of the fourth ventricle, which would explain the high incidence of dentate nucleus invasion.

Most interesting, the LS, if based solely on the postoperative damage pattern, is relatively low and, by itself, does not much affect determinations of the laterality of the tumor. The most likely explanation for this phenomenon may be that the postoperative damage pattern may include a few inadvertent lesions ("collateral damage"); therefore, the resultant LS is somewhat reduced by those. Nonetheless, we found the meticulous evaluation 
of the postoperative damage pattern to be helpful in understanding typical tumor locations.

On the basis of the observed involvement patterns, we propose that 4 apparent topographic phenotypes may be distinguished in WNT-subgroup medulloblastomas: 1) midline-intraventricular; 2) midline-extraventricular (centered on the cisterna magna with possible extensions toward the foramen magnum and/or the foramen of Magendie); 3) off-midline-intraventricular (centered on the foramen of Luschka with systematic extension to the fourth ventricle and optional extension into the $\mathrm{CP}$ angle cistern); and 4) off-midline-extraventricular (centered on the CP angle cistern, with more-or-less involvement of the foramen of Luschka). The extreme forms in each subtype are fairly straightforward, but overall, these phenotypic subtypes exhibit spatial overlaps, representing a continuum rather than 4 distinct categories. We have reason to believe that "midline" tumors (subtypes A and B) in the WNT-subgroup are actually paramedian (ie, close to the midline but not originating from the vermis) as opposed to the usually more lateralized SHH-subgroup tumors. Given that SHH-subgroup medulloblastomas are usually hemispheric (with few originating from the vermis) and typically extraventricular, and if WNT-subgroup medulloblastomas are indeed paramedian, then the only true midline-intraventricular tumors may be group 3 and 4 medulloblastomas. Further studies are needed to examine this hypothesis.

The incidence of PICA territory infarction in our patient cohort was high. The infarctions did not include the retro-olivary area; therefore, we speculate that the increased surgical risk to these patients may have to do with the often-suggested possible engulfment of the artery in its retromedullary course. We speculate that WNT-subgroup medulloblastomas may be associated with an increased risk for PICA infarction at surgery.

The laterality in conjunction with the dominantly extraparenchymal location of WNT-subgroup medulloblastomas would suggest that these tumors originate from mutant precursor cells on one side of the lower rhombic lip that fail to migrate along the anterior precerebellar extramural migratory stream. As a function of other modulating factors, these precursors may subsequently undergo neoplastic transformation and spread from the foramen of Luschka toward adjacent CSF spaces along the surface of the cerebellum and brain stem. This scenario implies that WNT-subgroup medulloblastomas are not cerebellar tumors.

We found that in midline-intraventricular (subtype A) WNT medulloblastomas, an obvious laterality is usually difficult to recognize at preoperative diagnostic imaging evaluation, complicating the differential diagnosis between these and other midline-intraventricular tumors, including group 3 and 4 medulloblastomas, some ependymomas, and choroid plexus tumors. Conversely, a tumor with DWI evidence of hypercellularity in any of the other 3 locations (subtypes B-D) may be more suggestive of WNT-subgroup medulloblastoma, but overlaps may occur. The infravermian (subtype B) tumors are rare but fairly characteristic, as are tumors with a clearly unilateral foramen of Luschka epicenter (subtype C). These location subtypes, however, may overlap with the typically hemispheric SHH-subgroup medulloblastomas (if those are inferomedial) and with some of the posterior fossa ependymomas. The CP angle cistern is also a well-known location for atypical teratoid rhabdoid tumors, complicating the initial imaging differential diagnosis of subtype D WNT-subgroup medulloblastomas; however, the biologic behavior and prognosis of the 2 are different. Bilateral involvement of the foramina of Luschka and of the CP angle cisterns is common in ependymoma and is rare in WNT-subgroup medulloblastoma. Choroid plexus tumors arising from the fourth ventricle are often midline-intraventricular but may be lateralized to involve the foramen of Luschka; however, their DWI appearance usually differs from that of embryonal tumors.

\section{CONCLUSIONS}

The spatial distribution of WNT-subgroup medulloblastomas in the posterior fossa appears to be in concordance with our current understanding of the embryologic origins of this tumor subtype. Our results contribute to the growing body of data showing the potential of "imaging genomics" for use in research and in the diagnostic imaging of certain genetically determined pathologies of the central nervous system.

Disclosures: Zoltan Patay—RELATED: Grant: National Institutes of Health, * American Lebanese Syrian Associated Charities, ${ }^{\star}$ Comments: St. Jude Children's Research Hospital is a Comprehensive Cancer Center receiving grant support from the $\mathrm{Na}$ tional Cancer Institute. The American Lebanese Syrian Associated Charities is the fundraising arm of St. Jude Children's Research Hospital. St. Jude Children's Research Hospital received support from the United States National Institutes of Health Cancer Center Support grant P30 CA21765 and the American Lebanese Syrian Associated Charities while the author participated in this research; UNRELATED: Consultancy: Guerbet, Comments: one-time consultancy in 2013; Grants/Grants Pending: Pediatric Brain Tumor Consortium, Comments: salary support from the Pediatric Brain Tumor Consortium; Travel/Accommodations/Meeting Expenses Unrelated to Activities Listed: Italian Society of Pediatric Neuroradiology (2014), European Course of MRI (2012-14), Kuwait Radiology Society (2013), Sao Paolo Radiology Society (2013), Hungarian Neuroradiology Society (2013), Indian Neuroradiology Society (2013), Arab Radiology Congress (2014), International Radiology Congress (2014), Symposium Neuroradiologicum (2014), Comments: travel and hotel reimbursement for giving invited educational lectures. David W. Ellison-UNRELATED: Patents (planned, pending, or issued): The patent is held for the immunophenotyping of medulloblastomas, but no payments are received. *Money paid to the institution.

\section{REFERENCES}

1. Northcott PA, Korshunov A, Witt H, et al. Medulloblastoma comprises four distinct molecular variants. J Clin Oncol 2011;29: 1408-14 CrossRef Medline

2. Northcott PA, Dubuc AM, Pfister S, et al. Molecular subgroups of medulloblastoma. Expert Rev Neurother 2012;12:871-84 CrossRef Medline

3. Northcott PA, Jones DT, Kool M, et al. Medulloblastomics: the end of the beginning. Nat Rev Cancer 2012;12:818-34 CrossRef Medline

4. Kool M, Korshunov A, Remke M, et al. Molecular subgroups of medulloblastoma: an international meta-analysis of transcriptome, genetic aberrations, and clinical data of WNT, SHH, group 3, and group 4 medulloblastomas. Acta Neuropathol 2012;123:473-84 CrossRef Medline

5. Ellison DW, Dalton J, Kocak M, et al. Medulloblastoma: clinicopathological correlates of SHH, WNT, and non-SHH/WNT molecular subgroups. Acta Neuropathol 2011;121:381-96 CrossRef Medline

6. Ellison DW, Kocak M, Dalton J, et al. Definition of disease-risk stratification groups in childhood medulloblastoma using combined clinical, pathologic, and molecular variables. J Clin Oncol 2011;29: 1400-07 CrossRef Medline

7. Ellison DW. Childhood medulloblastoma: novel approaches to the classification of a heterogeneous disease. Acta Neuropathol 2010; 120:305-16 CrossRef Medline

8. Ramaswamy V, Remke M, Bouffet E, et al. Recurrence patterns 
across medulloblastoma subgroups: an integrated clinical and molecular analysis. Lancet Oncol 2013;14:1200-07 CrossRef Medline

9. Gibson P, Tong Y, Robinson G, et al. Subtypes of medulloblastoma have distinct developmental origins. Nature 2010;468:1095-99 CrossRef Medline

10. Cicchetti DV. Guidelines, criteria, and rules of thumb for evaluating normed and standardized assessment instruments in psychology. Psychol Assess 1994;6:284-90 CrossRef

11. Landis JR, Koch GG. The measurement of observer agreement for categorical data. Biometrics 1977;33:159-74 CrossRef Medline

12. Teo WY, Shen J, Su JM, et al. Implications of tumor location on subtypes of medulloblastoma. Pediatr Blood Cancer 2013;60: 1408-10 CrossRef Medline

13. Ellison DW, Onilude OE, Lindsey JC, et al; United Kingdom Children's Cancer Study Group Brain Tumour Committee. $\boldsymbol{\beta}$-Catenin status predicts a favorable outcome in childhood medulloblastoma: the United Kingdom Children's Cancer Study Group Brain Tumour Committee. J Clin Oncol 2005;23:7951-57 CrossRef Medline

14. Mueller DP, Moore SA, Sato Y, et al. MRI spectrum of medulloblastoma. Clin Imaging 1992;16:250-55 CrossRef Medline

15. Eran A, Ozturk A, Aygun N, et al. Medulloblastoma: atypical CT and MRI findings in children. Pediatr Radiol 2010;40:1254-62 CrossRef Medline

16. Fruehwald-Pallamar J, Puchner SB, Rossi A, et al. Magnetic resonance imaging spectrum of medulloblastoma. Neuroradiology 2011; 53:387-96 CrossRef Medline

17. Yeom KW, Mobley BC, Lober RM, et al. Distinctive MRI features of pediatric medulloblastoma subtypes. AJR Am J Roentgenol 2013; 200:895-903 CrossRef Medline

18. Perreault S, Ramaswamy V, Achrol AS, et al. MRI surrogates for molecular subgroups of medulloblastoma. AJNR Am J Neuroradiol 2014;35:1263-69 CrossRef Medline 